Integrating Eq. (13) between -1 and +1 gives another relation between the $c_{i}$

$$
c_{0}(1-\ln 2) I_{1}(k)-c_{1} I_{0}(k)=0
$$

so that, solving for $c_{0}$ and $c_{1}$,

$$
c_{0}=-\frac{I_{0}(k)}{(\ln 2) I_{0}(k)+k I_{1}(k)}, \quad c_{1}=-\frac{(1-\ln 2) I_{1}(k)}{(\ln 2) I_{0}(k)+k I_{1}(k)}
$$

and the final solution may now be written down by substituting for $h(x)$ and the $c_{i}$ in Eq. (13). The special case of small $k$ is of interest; to the first order,

$$
f(x)=-\frac{1}{\pi \ln 2\left(1-x^{2}\right)^{1 / 2}}+k\left(1-\frac{1}{\ln 2}\right) \frac{x}{\pi\left(1-x^{2}\right)^{1 / 2}},
$$

where the first term is the well-known solution of Eq. (12) for $k=0$. The correction term bears a ratio to the first term of approximately $\left(\frac{1}{2} k x\right)$, which is of the expected magnitude.

To conclude, it may be remarked that to write out a formal solution is estimated to require about $2 n^{3 / 2}$ hours of labor for that case in which $P$ and $Q$ are $n$th order polynomials. Of course, once such a solution has been tabulated, it may be used for a number of kernels or parameters. Note also, as previously mentioned, the possibility of curvefitting. In particular, if a large number of terms are to be used, it may be worthwhile to set $P=1$ and represent the kernel behavior by $[1 n|x-t|+Q]$ alone; the $Q$ terms could then of course be absorbed directly into $g(x)$ and Eq. (3) used.

\title{
REFERENCES
}

1. N. Wiener and E. Hopf, Berlin Ber., Math. Phys. Klasse, 1931, p. 696

2. T. Carleman, Math. Z. 15, 111 (1922)

3. T. Carleman, Arkiv. for Mat., Astron., och Fysik 16, No. 26 (1922)

4. G. Latta, Tech. Rep. No. 32 of Appl. Math. and Stat. Lab., 1955, Stanford Univeraity, Calif.

5. N. Muskhelishvili, Singular integral equations, Transl. by J. R. M. Radok, Noordhof, Groningen, 1953

\section{PRIORITY ASSIGNMENT ON A WAITING LINE*}

\section{By S. A. DRESSIN" ${ }^{1}$ AND E. REICH ${ }^{2}$ (Santa Monica, California)}

Interest in queueing statistics when a priority system is involved is now widespread, especially in connection with communications applications. In this paper we consider a model described in the usual customer-counter terminology as follows.

Input. There are $r$ classes of customers, a particular class, labelled by the integer $p$, $(p=1,2, \ldots, r)$, being made up of customers of "priority" $p$. (Following an unfortunate, but standard, practice, priority $p_{1}$ will be said to be higher than priority $p_{2}$ if and only if $p_{1}<p_{2}$.) Customers of priority $p$ arrive as a Poisson process with interarrival-period distribution $\lambda_{p} e^{-\lambda_{p} x} d x, x>0$. Customers of different priorities arrive independently.

Queue-discipline. Once a customer's service has begun, it is permitted to proceed to completion. If the counter becomes empty, and there is at least one customer waiting,

*Received May 7, 1956.

${ }^{1}$ Major, U. S. M. C., U. S. Naval Postgraduate School, Monterey, Calif.

${ }^{2}$ Now at University of Minnesota. 
then a customer of the highest priority present or queue is admitted to the counter, and his service starts. Customers of equal priority are served first come, first served.

Service mechanism. The service period has the distribution $\mu e^{-\mu x} d x, x>0$. Service periods are mutually independent, and independent of input history and priority.

The above model will be recognized to be a specialization of one considered by Cobham [1]. It is reasonable in some applications, and has the mathematical virtue of yielding simpler results than more general queue disciplines. This paper extends previous work in that we compute conditional and unconditional distributions, rather than only means.

In what follows, we put.

$$
\sigma_{p}=\sum_{i=1}^{p} \lambda_{i}, \quad \sigma_{r}=\sigma .
$$

The system will be said to be in state $n$ if the total number of customers at the counter and on queue is $n(n=0,1,2, \cdots)$. At the same time, the system will be said to be in state $(n ; p), n=1,2, \cdots$, if there is a customer of arbitrary priority at the counter, and there are exactly $n-1$ customers, of priority $p$ or higher, on queue. State $(0 ; p)$ will be identified with state 0 .

System behavior with time. It is clear that the value of $n$ at time $t$ is the same as if we were dealing with the classical case $r=1$. The reason for this is that the particular queue discipline under consideration can be transformed to first come, first served, by merely reordering those customers on queue, in a manner not depending on their service times. If we continue this reasoning, applying it to the class of cutomers of priority $p$ or higher, we see that the following is true: For every fixed $p$, the vector $A=[k,(m ; p)]$ is a Markoffin function of time.

Let $P_{n}(t)=$ probability that the system is in state $n$ at time $t, P_{(n ; p)}(t)=$ probability that the system is in state $(n ; p)$ at time $t$. In view of what was said above, the differential equations for $P_{n}(t)$ are the well-known ones [4],

$$
\begin{aligned}
& \partial_{t} P_{0}(t)=-\sigma P_{0}(t)+\mu P_{1}(t), \\
& \partial_{t} P_{n}(t)=\sigma P_{n-1}(t)-(\sigma+\mu) P_{n}(t)+\mu P_{n+1}(t), \quad n=1,2, \cdots .
\end{aligned}
$$

Trivially,

$$
P_{(0 ; p)}(t)=P_{0}(t) .
$$

Let $h>0$ be a small quantity. Neglecting probabilities of order $h^{2}$, we can say that the system is in state $(1 ; p)$ at time $t=t_{0}$ if and only if at least one of the following mutually exclusive events has occurred.

(i) State at $t=t_{0}-h$ was $(1 ; p)$, no service ended since $t_{0}-h$, no customers of priority $p$ or higher arrived since $t_{0}-h$, or

(ii) the value of $A$ at time $t_{0}-h$ was $[k,(1 ; p)], k>1$, a service period ended since $t_{0}-h$, no customers of priority $p$ or higher arrived since $t_{0}-h$, or

(iii) state at $t_{0}-h$ was $(0 ; p)$, a customer of arbitrary priority arrived since $t_{0}-h$, or

(iv) state at $t_{0}-h$ was $(2 ; p)$, a service period ended since $t_{0}-h$, no customers of priority $p$ or higher arrived since $t_{0}-h$. Now, within terms of order $h^{2}$,

$$
\begin{aligned}
& \text { Prob (i) }=\left(1-\mu h-\sigma_{p} h\right) P_{(1 ; p)}\left(t_{0}-h\right), \\
& \text { Prob (ii) }=\mu h\left[P_{(1 ; p)}(t-h)-P_{1}(t-h)\right],
\end{aligned}
$$




$$
\begin{aligned}
& \text { Prob (iii) }=\sigma h P_{0}(t-h), \\
& \text { Prob (iv) }=\mu h P_{(2 ; p)}(t-h) .
\end{aligned}
$$

Hence

$$
\partial_{t} P_{(1 ; p)}(t)=\sigma P_{0}(t)-\mu P_{1}(t)-\sigma_{p} P_{(1 ; p)}(t)+\mu P_{(2 ; p)}(t) .
$$

Similarly,

$$
\partial_{l} P_{(n ; p)}(t)=\sigma_{p} P_{(n-1 ; p)}(t)-\left(\sigma_{p}+\mu\right) P_{(n ; p)}(t)+\mu P_{(n+1 ; p)}(t), \quad n=2,3, \cdots .
$$

Note that Eqs. (3)-(5) involve $P_{0}(t), P_{1}(t)$, as determined by Eqs. (1), (2). If $\sigma<\mu$ then the "equilibrium" solutions $P_{k}, P_{(k ; p)}$, of (1)-(5), for which

$$
\sum_{k=0}^{\infty} P_{k}=1=\sum_{k=0}^{\infty} P_{(k ; p)},
$$

are

$$
P_{k}=\left(\frac{\mu-\sigma}{\mu}\right)\left(\frac{\sigma}{\mu}\right)^{k}, \quad k=0,1,2, \cdots
$$

and, therefore

$$
P_{(0 ; p)}=\frac{\mu-\sigma}{\mu}, \quad P_{(k ; p)}=\frac{\left(\mu-\sigma_{p}\right) \sigma}{\mu \sigma_{p}}\left(\frac{\sigma_{p}}{\mu}\right)^{k}, \quad k=1,2, \cdots .
$$

If $\sigma>\mu>\sigma_{p}$, the appropriate values are

$$
\begin{gathered}
P_{k}=0, \quad(k=0,1,2, \cdots), \\
P_{(0 ; p)}=0, \quad P_{(k ; p)}=\left(\frac{\mu-\sigma_{p}}{\mu}\right)\left(\frac{\sigma_{p}}{\mu}\right)^{k-1}, \quad(k=1,2, \cdots) .
\end{gathered}
$$

Conditional queueing times. We now calculate the probability $f_{(n ; p)}(T) d T$ that a customer $C$ of priority $p$ will have to wait on queue for a duration of between $T$ and $T+d T$, conditional to the assumption that the system is in state $(n ; p)$ the instant before $C$ 's arrival. It is clear that the probability in question would not be altered if it were further conditioned relative to some aspect of the queue's history prior to $C$ 's arrival.

Of course

$$
f_{(0 ; p)}(T)=\delta(T-0), \quad p=1,2, \cdots, r .
$$

Also,

$$
\begin{aligned}
f_{(n ; 1)}(T)=n \text { fold convolution of } \mu e^{-\mu T}=\mu^{n} T^{n-1} e^{-\mu T} /(n-1) !, \\
\qquad T>0, \quad n=1,2, \cdots .
\end{aligned}
$$

By employing a simple device we can immediately write down $f_{(1 ; p)}(T), p>1$. If a customer $C$ of priority $p$ finds, on arrival, that the only customer delaying him is a customer at the counter, then $C$ 's queueing time $T$ depends on the additional service time of the customer at the counter, plus the service times of the customers of priority higher than $p$ who arrive while the customer at the counter is served, etc. $T$ evidently has the same distribution as the length of the busy period of the classical single-priority queue with input and service parameters $\sigma_{\nu-1}$ and $\mu$, respectively, that is, $[3,4]$, if 


$$
\begin{gathered}
\sigma_{p-1}<\mu, \quad f_{(1 ; p)}(T)=\left(\mu / \sigma_{p-1}\right)^{1 / 2} T^{-1} \exp \left[-\left(\sigma_{p-1}+\mu\right) T\right] I_{1}\left(2 \sigma_{p-1}^{1 / 2} \mu^{1 / 2} T\right), \\
T>0, \quad p=2,3, \cdots,
\end{gathered}
$$

where $I_{n}$ is the modified Bessel function of order $n$. If the system is in state $(n ; p) n>1$, $p>1$, the instant before $C$ 's arrival, say at $t=0$, then $T$ is unaffected if we reduce the priorities of those customers of priority higher than $p$, who are on queue at $t=0$, to $p$. Assume this reduction is carried out. Let $C^{*}$ denote that customer of priority $p$ who is directly in front of $C$, and let $T^{*}$ denote the remaining queueing time of $C^{*}$, from $t=0$ on. $T^{*}$ has the distribution $f_{(n-1 ; p)}(x) d x$. At $t=T^{*}, C^{*}$ 's service starts, and $C$ is first on line in front of the counter. Hence the variable $T-T^{*}$ has distribution $f_{(1 ; p)}(x) d x$, and is independent of $T^{*}$. Consequently, $f_{(n ; p)}(x)$ is the convolution of $f_{(n-1 ; p)}(x)$ and $f_{(1 ; p)}(x)$; that is,

$f_{(n ; p)}(T)=n$ fold convolution of $f_{(1 ; p)}(T)$

$$
\begin{aligned}
& =n\left(\mu / \sigma_{p-1}\right)^{n / 2} T^{-1} \exp \left[-\left(\sigma_{p-1}+\mu\right) T\right] I_{n}\left(2 \sigma_{p-1}^{1 / 2} \mu^{1 / 2} T\right), \\
& T>0, \quad n=1,2, \cdots, \quad p=2,3, \cdots .^{3}
\end{aligned}
$$

Unconditional queueing times. Suppose test-customer of priority $p$ enters the system at time $t$. The probability that he will have to wait on queue for a period between $T$ and $T+d T$ is

$$
F_{p}(T ; t) d T=\sum_{k=0}^{\omega} P_{(k ; p)}(t) f_{(k ; p)}(T) d T .
$$

In "equilibirum", we have, by (7)-(10), if $\sigma<\mu$,

$$
E\left\{e^{-s T}\right\}=1-(\sigma / \mu)+2(\sigma / \mu)\left(\mu-\sigma_{p}\right) Q^{-1}
$$

where

$$
\begin{gathered}
Q=s+\sigma_{p-1}+\mu-2 \sigma_{p}+\left(s+\sigma_{p-1}+\mu+2 \sigma_{p-1}^{1 / 2} \mu^{1 / 2}\right)^{1 / 2}\left(s+\sigma_{p-1}+\mu-2 \sigma_{p-1}^{1 / 2} \mu^{1 / 2}\right)^{1 / 2}, \\
\sigma_{0}=0 .
\end{gathered}
$$

An analogous expression is obtained when $\sigma>\mu>\sigma_{\nu}$.

Note that Eq. (11) gives, for $p=1$,

$$
\text { Prob }\{T \leq x\}=1-(\sigma / \mu) e^{-\left(\mu-\lambda_{1}\right) x}, \quad x \geq 0 .
$$

\section{REFERENCES}

1. A. Cobham, Priority assignment in waiting line problems, J. Opns. Res. Soc. Am. 2, 70-76 (1954)

2. J. L. Holley, Waiting line subject to priorities, J. Opns. Res. Soc. Am. 2, 341-343 (1954)

3. D. G. Kendall, Some problems in the theory of queues, J. Roy. Stat. Soc. B 13, 151-184 (1951)

4. N. T. J. Bailey, A continuous time treatment of a simple queue using generating functions, J. Roy. Stat. Soc. B 16, 288-291 (1954)

5. D. G. Kendall, Stochastic processes occurring in the theory of queues and their analysis by the method of the imbedded Markov chain, Ann. Math. Stat. 24, 338-360 (1953)

6. W. Ledermann and G. E. H. Reuter, Spectral theory for the differential equations of simple birth and death processes, Phil. Trans. Roy. Soc. London A 246, 321-369 (1954)

${ }^{3}$ This result can also be obtained by using Eq. (4.14) of [6]. 